\title{
On the properties of the intensity-curvature measurement approaches: The classic-curvature and the intensity-curvature functional
}

\author{
Carlo Ciulla*1, Dimitar Veljanovski ${ }^{2}$, Ustijana Rechkoska Shikoska ${ }^{1}$, Filip A. Risteski ${ }^{2}$ \\ ${ }^{1}$ University of Information Science \& Technology, Ohrid, Macedonia \\ ${ }^{2}$ Department of Radiology, General Hospital 8-mi Septemvri, Skopje, Macedonia
}

Received: October 15, 2015

DOI: $10.5430 /$ jst.v6n1p38
Accepted: December 2, $2015 \quad$ Online Published: December 20, 2015

URL: http://dx.doi.org/10.5430/jst.v6n1p38

\begin{abstract}
Objective: This paper expands on the basis of previous research confirming through empirical evidence both meaning and nature of the classic-curvature and the intensity-curvature functional images.

Methods: Eleven subjects affected by human brain tumors were scanned using Magnetic Resonance Imaging (MRI). Twodimensional MRI images were selected to study the subjects using the intensity-curvature measurement approaches called: classic-curvature and intensity-curvature functional. The bivariate cubic polynomial and the bivariate linear function were fitted to the MRI images in order to compute the classic-curvature and the intensity-curvature functional respectively.

Results: This work shows one novelty related to the use of the two intensity-curvature measurement approaches when furthering the study of tumors detected with MRI. The novelty is the highlight of the accumulation of the contrast agent through the visually perceptible third dimension perpendicular to the imaging plane.

Conclusions: The meaning and the nature of the aforementioned intensity-curvature measurement approaches is suggested to be the high pass filtered MRI signal.
\end{abstract}

Key Words: Intensity-curvature measurement approaches, Classic-curvature, Intensity-curvature functional, Visually perceptible third dimension, Magnetic resonance imaging, Human brain, Tumor

\section{INTRODUCTION}

\subsection{The literature and the theme of the work}

Usually, the most immediate source of additional information to the T1-weighted MRI is the contrast enhanced T1-weighted MRI. Moreover, techniques like: T2weighted MRI ${ }^{[1]}$ Perfusion Weighted Imaging (PWI), ${ }^{[2]}$ Diffusion Weighted Imaging (DWI), ${ }^{[3]}$ Diffusion Tensor Imaging (DTI) ${ }^{[4]}$ Susceptibility Weighted Imaging (SWI),${ }^{[5]}$ Magnetic Resonance Spectroscopy (MRS), ${ }^{[6,7]}$ Magnetic Resonance Angiography (MRA) (including FLASH MRI and Magnetic resonance venography), ${ }^{[6]}$ Functional MRI (fMRI), ${ }^{[8-10]}$ Intraoperative MRI, ${ }^{[9]}$ Magnetic Resonance guided focused ultrasound, ${ }^{[11]}$ Multinuclear imaging, ${ }^{[12]}$ and Molecular imaging by MRI, ${ }^{[13]}$ are all in support of the T1weighted MRI for what pertains the collection of supplemental information about the case study.

This paper furthers the quest for supplemental information about human brain tumors imaged with MRI. The supplemental information is provided through the use of two intensitycurvature measurement approaches ${ }^{[14]}$ named: 1$)$ the classic-

\footnotetext{
*Correspondence: Carlo Ciulla; Email: carlo.ciulla@uist.edu.mk, cxc2728@njit.edu; Address: University of Information Science \& Technology, Partizanska B.B., 6000 Ohrid, Macedonia.
} 
curvature (CC) and 2) the intensity-curvature functional (ICF). Recent research has reported on the capability of the classic-curvature and the intensity-curvature functional to act as the MRI high pass filtered signal, ${ }^{[15]}$ and the mathematical model functions fitted to the MRI data were: 1) the bivariate cubic Lagrange polynomial, 2) the bivariate linear function, 3) the univariate cubic Lagrange polynomial and 4) the univariate cubic B-spline. The use of the aforementioned intensity-curvature measurement approaches is similar in this paper because the classic-curvature and the intensity-curvature functional are used as filter masks capable to bring the two-dimensional MRI images of the human brain tumors into two additional domains: the filtered MRI images. However, this paper extends from the previously mentioned research because investigates further the filtering behavior of the $\mathrm{CC}$ images when using a different model function: the bivariate cubic polynomial. ${ }^{[16]}$

The visually perceptible third dimension of both $\mathrm{CC}$ and ICF images is defined as that dimension perceptible along the axis perpendicular to the 2D imaging plane. ${ }^{[17,18]}$ However the implications, and the true meaning and nature of the visually perceptible third dimension were not fully clarified. Thus, this paper follows through earlier research ${ }^{[15,17,18]}$ because reports additional findings related to the visually perceptible third dimension of both $\mathrm{CC}$ and ICF images. The findings are related to the capability of the $\mathrm{CC}$ and the ICF to highlight the accumulation of fluids in the human brain when using a variety of MRI modalities: T1-weighted MRI, contrast enhanced T1-weighted MRI, T2-weighted MRI and Fluid Attenuated Inversion Recovery (FLAIR) MRI. ${ }^{[19]}$

And using the bivariate cubic polynomial ${ }^{[18]}$ function this paper presents evidence in support to the hypothesis that the visually perceptible third dimension is clearly shown in CC images calculated from contrast enhanced T1-weighted MRI images. The difference that this paper proposes respect to previous research ${ }^{[17]}$ is the focus on the study of the contrast enhanced T1-weighted MRI because of the capability of the $\mathrm{CC}$ image to highlight the regions of the brain and the tumor where the contrast agent is accumulated. Moreover, as it will be presented in this paper, the use of the $\mathrm{CC}$ images showing the visually perceptible third dimension is to filter the MRI.

Additionally, it is important to acknowledge that the methodology employed in this paper is unreported. The methodology uses the comparison of the $\mathrm{CC}$ and the ICF images with the high pass filtered MRI signal.

\subsection{The research question and the two intensity- curvature measurement approaches}

The research question addressed in this paper consists in the elucidation of the significance of the visually percepti- ble third dimension shown by the classic-curvature and the intensity-curvature functional images, which is observed perpendicularly to the image in 2D pathological human brain MRI images. ${ }^{[17,18]}$ The significance of the visually perceptible third dimension consists of two aspects: 1) the implication, and 2) the true meaning and nature.

The implication is the highlight of the accumulation of contrast agent in MRI images. The true meaning and nature of the CC and ICF is studied in this paper expanding the results earlier reported ${ }^{[15]}$ and it is suggested to be the high pass filtered MRI signal. In this paper, the $\mathrm{CC}$ and the ICF were calculated when fitting to the MRI data the bivariate cubic polynomial and the bivariate linear model function respectively.

The aforementioned aspects find application in Magnetic Resonance Imaging of the human brain in pathological states and more specifically within the domain of the study of human brain tumors.

\section{SUbJECTS AND DATA}

\subsection{Subjects}

The subjects were the same as those studied earlier. ${ }^{[14,18]}$ The subjects were: 1) a 40 years old female diagnosed with brain metastases, 2) a 77 years old male diagnosed with glioblastoma multiforme, 3 ) a 33 years old female diagnosed with intra-ventricular brain tumor, 4) a 64 years old male diagnosed with oligodendroglioma, 5) a 72 years old male diagnosed with brain metastases, 6) a 55 years old male diagnosed with brain metastases from pulmonary cancer, 7) a 41 years old female diagnosed with anaplastic oligodendroglioma, 8) a 38 years old female diagnosed with meningioma, 9) a 37 years old female diagnosed with cystic glioblastoma, 10) a 44 years old female diagnosed with glioblastoma, and 11) a 42 years old male diagnosed with tumor in the brain with intra-ventricular extension. The MRI scanning procedures were conducted in compliance with the ethical standards set by the General Hospital 8-mi Septemvri, Skopje-Macedonia, which is the institution where the patients were diagnosed.

\subsection{Data}

Table 1 reports on the MRI data employed in this research in reference to Figures 1-12 of the manuscript. The twodimensional MRI images used in this research were extracted from three-dimensional MRI volumes. The recording parameters and 2D image resolution are the same as those reported earlier, ${ }^{[14]}$ however, the two-dimensional MRI images used in this research are not the same as those analyzed earlier (except for two cases). ${ }^{[14,18]}$ The post-processing of the 2D MRI images was conducted with the analysis of the following images: 1) the CC calculated when fitting to the MRI 
data the bivariate cubic polynomial given in chapter $1,^{[16]}$ and 2) the intensity-curvature functional calculated when fitting to the MRI data the bivariate linear function. ${ }^{[17]}$ Data

Table 1. Data employed in this research in reference to the figures presented in the manuscript

\begin{tabular}{|c|c|c|c|c|}
\hline Figure/Data & Pathology & MRI Imaging Modality & $\begin{array}{l}\text { Pixel matrix size; } \\
\text { Pixel size }\end{array}$ & $\begin{array}{l}\text { Math Model; Formulae; } \\
\text { Re-sampling coordinate }\end{array}$ \\
\hline Figure 1 & brain metastases & $\begin{array}{l}\text { T2-weighted MRI, TE = } 96 \\
\text { msec, } \mathrm{TR}=5,050 \mathrm{msec}\end{array}$ & $\begin{array}{l}262 \times 320 ; \\
0.78 \mathrm{~mm} \times 0.78 \mathrm{~mm}\end{array}$ & $\begin{array}{l}\text { bivariate cubic polynomial, } \\
\text { bivariate linear; CC, ICF; } \\
(\mathrm{x}, \mathrm{y})=(0.5 \mathrm{~mm}, 0.5 \mathrm{~mm})\end{array}$ \\
\hline Figure 2 & brain metastases & $\begin{array}{l}\text { T2-weighted pulse sequence } \\
\text { MRI, TE = } 96 \mathrm{msec} \text {, } \\
\text { TR = 4,720 msec }\end{array}$ & $\begin{array}{l}262 \times 320 ; \\
0.78 \mathrm{~mm} \times 0.78 \mathrm{~mm}\end{array}$ & $\begin{array}{l}\text { bivariate cubic polynomial, } \\
\text { bivariate linear; CC, ICF; } \\
(\mathrm{x}, \mathrm{y})=(0.5 \mathrm{~mm}, 0.5 \mathrm{~mm})\end{array}$ \\
\hline Figure 3 & brain metastases & $\begin{array}{l}\text { contrast enhanced } \\
\text { T1-weigthed MRI; TE = } 9 \\
\text { msec, TR = } 550 \text { msec }\end{array}$ & $\begin{array}{l}512 \times 512 ; \\
0.45 \mathrm{~mm} \times 0.45 \mathrm{~mm}\end{array}$ & $\begin{array}{l}\text { bivariate cubic polynomial, } \\
\text { bivariate linear; CC, ICF; } \\
(\mathrm{x}, \mathrm{y})=(0.25 \mathrm{~mm}, 0.25 \mathrm{~mm})\end{array}$ \\
\hline Figure 4 & $\begin{array}{l}\text { glioblastoma } \\
\text { multiforme }\end{array}$ & $\begin{array}{l}\text { T2-weighted MRI; TE = } 96 \\
\text { msec, TR = 5,050 msec }\end{array}$ & $\begin{array}{l}262 \times 320 ; \\
0.72 \mathrm{~mm} \times 0.72 \mathrm{~mm}\end{array}$ & $\begin{array}{l}\text { bivariate cubic polynomial, } \\
\text { bivariate linear; CC, ICF; } \\
(\mathrm{x}, \mathrm{y})=(0.35 \mathrm{~mm}, 0.35 \mathrm{~mm})\end{array}$ \\
\hline Figure 5 & $\begin{array}{l}\text { glioblastoma } \\
\text { multiforme }\end{array}$ & $\begin{array}{l}\text { T2-weighted MRI; TE = } 109 \\
\text { msec, TR =750 msec }\end{array}$ & $\begin{array}{l}512 \times 512 ; \\
0.45 \mathrm{~mm} \times 0.45 \mathrm{~mm}\end{array}$ & $\begin{array}{l}\text { bivariate cubic polynomial, } \\
\text { bivariate linear; CC, ICF; } \\
(\mathrm{x}, \mathrm{y})=(0.25 \mathrm{~mm}, 0.25 \mathrm{~mm})\end{array}$ \\
\hline Figure 6 & $\begin{array}{l}\text { glioblastoma } \\
\text { multiforme }\end{array}$ & $\begin{array}{l}\text { contrast enhanced T1- } \\
\text { weighted MRI; TE = } 8.7 \mathrm{msec} \text {, } \\
\mathrm{TR}=680 \mathrm{msec}\end{array}$ & $\begin{array}{l}512 \times 512 ; \\
0.48 \mathrm{~mm} \times 0.48 \mathrm{~mm}\end{array}$ & $\begin{array}{l}\text { bivariate cubic polynomial, } \\
\text { bivariate linear; CC, ICF; } \\
(\mathrm{x}, \mathrm{y})=(0.24 \mathrm{~mm}, 0.24 \mathrm{~mm})\end{array}$ \\
\hline Figure 7 & $\begin{array}{l}\text { glioblastoma } \\
\text { multiforme }\end{array}$ & $\begin{array}{l}\text { contrast enhanced T1- } \\
\text { weighted MRI; TE = } 8.7 \mathrm{msec} \text {, } \\
\mathrm{TR}=680 \mathrm{msec}\end{array}$ & $\begin{array}{l}512 \times 512 ; \\
0.48 \mathrm{~mm} \times 0.48 \mathrm{~mm}\end{array}$ & $\begin{array}{l}\text { bivariate cubic polynomial, } \\
\text { bivariate linear; CC, ICF; } \\
(\mathrm{x}, \mathrm{y})=(0.24 \mathrm{~mm}, 0.24 \mathrm{~mm})\end{array}$ \\
\hline Figure 8 & $\begin{array}{l}\text { intra-ventricular } \\
\text { brain tumor }\end{array}$ & $\begin{array}{l}\text { FLAIR; TE = } 84 \text { msec, } \\
\text { TR = 9,000 msec }\end{array}$ & $\begin{array}{l}260 \times 320 ; \\
0.78 \mathrm{~mm} \times 0.78 \mathrm{~mm}\end{array}$ & $\begin{array}{l}\text { bivariate cubic polynomial, } \\
\text { bivariate linear; CC, ICF; } \\
(\mathrm{x}, \mathrm{y})=(0.35 \mathrm{~mm}, 0.35 \mathrm{~mm})\end{array}$ \\
\hline Figure 9 & $\begin{array}{l}\text { intra-ventricular } \\
\text { brain tumor }\end{array}$ & $\begin{array}{l}\text { T1-weighted MRI; TE = } 8.7 \\
\text { msec, TR = } 550 \text { msec }\end{array}$ & $\begin{array}{l}512 \times 512 ; \\
0.45 \mathrm{~mm} \times 0.45 \mathrm{~mm}\end{array}$ & $\begin{array}{l}\text { bivariate cubic polynomial, } \\
\text { bivariate linear; CC, ICF; } \\
(\mathrm{x}, \mathrm{y})=(0.25 \mathrm{~mm}, 0.25 \mathrm{~mm})\end{array}$ \\
\hline Figure 10 & glioblastoma & $\begin{array}{l}\text { FLAIR; TE = } 84 \text { msec, } \\
\text { TR = 9,000 msec }\end{array}$ & $\begin{array}{l}260 \times 320 ; \\
1.00 \mathrm{~mm} \times 1.00 \mathrm{~mm}\end{array}$ & $\begin{array}{l}\text { bivariate linear; ICF; } \\
(\mathrm{x}, \mathrm{y})=(0.5 \mathrm{~mm}, 0.5 \mathrm{~mm})\end{array}$ \\
\hline Figure 11 & $\begin{array}{l}\text { metastases from } \\
\text { pulmonary cancer }\end{array}$ & $\begin{array}{l}\text { T2-weighted MRI; TE = } 96 \\
\text { msec, TR = 4,720 msec }\end{array}$ & $\begin{array}{l}262 \times 320 ; \\
0.78 \mathrm{~mm} \times 0.78 \mathrm{~mm}\end{array}$ & $\begin{array}{l}\text { bivariate cubic polynomial, } \\
\text { bivariate linear; CC, ICF; } \\
(x, y)=(0.5 \mathrm{~mm}, 0.5 \mathrm{~mm})\end{array}$ \\
\hline Figure 12 & $\begin{array}{l}\text { Various pathologies: } \\
\text { see figure }\end{array}$ & $\begin{array}{l}\text { T1-weighted MRI; contrast } \\
\text { enhanced T1-weighted MRI; } \\
\text { TR and TE: see figure }\end{array}$ & $\begin{array}{l}512 \times 512 ; 260 \times 320 \\
1.00 \mathrm{~mm} \times 1.00 \mathrm{~mm}\end{array}$ & $\begin{array}{l}\text { bivariate cubic polynomial, } \\
\mathrm{CC} ;(\mathrm{x}, \mathrm{y})=(0.5 \mathrm{~mm}, 0.5 \mathrm{~mm})\end{array}$ \\
\hline
\end{tabular}

Note. From the left most column to the right most column: the figure number, the pathology, the MRI modality, the image resolution, the polynomial functions, and the intensity-curvature measurement approaches calculated from the MRI: (i) the classic-curvature (CC) and (ii) the intensity-curvature functional (ICF). FLAIR is the abbreviation of Fluid Attenuated Inversion Recovery ${ }^{[19]}$, which is an MRI imaging modality used to suppress fluids in the human brain.

\section{THEORY}

\section{The methodology}

The classic-curvature and the intensity-curvature functional of a signal-image are calculated in this section of the paper. A model function is fitted to the signal. The classiccurvature $^{[16]}$ is defined as the sum of all of the second-order derivatives of the Hessian of the model function. To calculate the intensity-curvature functional is necessary to calculate from eleven subjects were analyzed and post-processed in order to investigate the research question of the paper. 
the product between: 1) the value of the signal calculated with the model function at the intra-pixel re-sampling coordinate of interest, and 2) the classic-curvature calculated at such re-sampling coordinate. The ICF is thus defined by the ratio of the two intensity curvature terms: $\Delta \mathrm{E}(\mathrm{x}, \mathrm{y})=$ $\mathrm{E}_{0} / \mathrm{E}_{I N} \cdot{ }^{[16]}$ In this paper, the $\mathrm{CC}$ is calculated when fitting the bivariate cubic polynomial to the MRI data as previously illustrated, ${ }^{[16]}$ whereas the math process utilized to calculate the ICF uses the bivariate linear function fitted to the MRI data. $^{[17]}$

Let us consider the bivariate cubic polynomial ${ }^{[14]}$ as per equation (1):

$$
\begin{aligned}
& f(x, y)=f(0,0)+[f(1,0)-f(0,0)]\left(a x^{2}+y\right)+ \\
& {[f(0,1)-f(0,0)]\left(b y^{2}-x\right)+[f(1,1)+f(0,0)} \\
& -f(0,1)-f(1,0)]\left(a x^{2} y+b y^{2} x\right)
\end{aligned}
$$

Where $f(1,0), f(0,1)$ and $f(1,1)$ are the pixels in the neighborhood of $f(0,0)$, which is the pixel for which the classiccurvature has to be calculated.

Let us posit:

$$
\begin{aligned}
& \varnothing_{a}=[f(1,0)-f(0,0)] \\
& \varnothing_{b}=[f(0,1)-f(0,0)] \\
& \varnothing_{a b}=[f(1,1)+f(0,0)-f(0,1)-f(1,0)]
\end{aligned}
$$

Thus:

$$
\begin{aligned}
& f(x, y)=f(0,0)+\varnothing_{a}\left(a x^{2}+y\right)+ \\
& \varnothing_{b}\left(b y^{2}-x\right)+\varnothing_{a b}\left(a x^{2} y+b y^{2} x\right)
\end{aligned}
$$

The first and second order partial derivatives of $f(x, y)$ are calculated in equations (6) through (11):

$$
\begin{aligned}
& \frac{\partial f(x, y)}{\partial x}=\varnothing_{a}(2 a x)-\varnothing_{b}+\varnothing_{a b}\left(2 a x y+b y^{2}\right) \\
& \frac{\partial f(x, y)}{\partial y}=\varnothing_{a}+\varnothing_{b}(2 b y)+\varnothing_{a b}\left(a x^{2}+2 b y x\right) \\
& \frac{\partial^{2} f(x, y)}{\partial x^{2}}=\varnothing_{a}(2 a)+\varnothing_{a b}(2 a y) \\
& \frac{\partial^{2} f(x, y)}{\partial y^{2}}=\varnothing_{b}(2 b)+\varnothing_{a b}(2 b x) \\
& \frac{\partial^{2} f(x, y)}{\partial x \partial y}=\varnothing_{a b}(2 a x+2 b y)
\end{aligned}
$$

$$
\frac{\partial^{2} f(x, y)}{\partial y \partial x}=\varnothing_{a b}(2 a x+2 b y)
$$

The classic-curvature of the bivariate cubic polynomial $f(x, y)$ is:

$$
\begin{aligned}
& Y_{C}(x, y)=\left(\frac{\partial^{2} f(x, y)}{\partial x^{2}}+\frac{\partial^{2} f(x, y)}{\partial y^{2}}+\frac{\partial^{2} f(x, y)}{\partial x \partial y}+\right. \\
& \left.\frac{\partial^{2} f(x, y)}{\partial y \partial x}\right)=\varnothing_{a}(2 a)+\varnothing_{a b}(2 a y)+\varnothing_{b}(2 b)+ \\
& \varnothing_{a b}(2 b x)+2 \varnothing_{a b}(2 a x+2 b y)
\end{aligned}
$$

Let $h(x, y)$ be the bivariate linear interpolation function which takes the form:

$$
\begin{aligned}
& h(x, y)=f(0,0)+x[f(1,0)-f(0,0)]+y[f(0,1)- \\
& f(0,0)]+x y[f(1,1)+f(0,0)-f(0,1)-f(1,0)]
\end{aligned}
$$

Where $f(1,0), f(0,1)$ and $f(1,1)$ are the pixels in the neighborhood of $f(0,0)$, which is the pixel for which the intensitycurvature functional is calculated. Let us posit:

$$
\theta_{x}=[f(1,0)-f(0,0)]
$$

$$
\theta_{y}=[f(0,1)-f(0,0)]
$$

$$
\omega_{f}=[f(1,1)+f(0,0)-f(0,1)-f(1,0)]
$$

It follows that:

$$
h(x, y)=f(0,0)+x \theta_{x}+y \theta_{y}+x y \omega_{f}
$$

The intensity-curvature term of $h(x, y)$ at the grid point $(x, y)$ $=(0,0)$ is called intensity-curvature term before interpolation and is calculated as:

$$
\begin{aligned}
& E_{0}=E_{0}(x, y)=\int_{0}^{x} \int_{0}^{y} f(0,0) \cdot\left(\frac{\partial^{2} h(x, y)}{\partial x^{2}}+\right. \\
& \left.\frac{\partial^{2} h(x, y)}{\partial y^{2}}+\frac{\partial^{2} h(x, y)}{\partial x \partial y}+\frac{\partial^{2} h(x, y)}{\partial y \partial x}\right)_{(0,0)} d x d y \\
& =\int_{0}^{x} \int_{0}^{y} f(0,0) 2 \omega_{f} d x d y=f(0,0) 2 x y \omega_{f}
\end{aligned}
$$

The intensity-curvature term of $h(x, y)$ at any intra-pixel location $(x, y)$ is called intensity-curvature term after interpolation and is defined as:

$$
\begin{aligned}
& E_{I N}=E_{I N}(x, y)=\int_{0}^{x} \int_{0}^{y} h(x, y) \cdot\left(\frac{\partial^{2} h(x, y)}{\partial x^{2}}+\right. \\
& \left.\frac{\partial^{2} h(x, y)}{\partial y^{2}}+\frac{\partial^{2} h(x, y)}{\partial x \partial y}+\frac{\partial^{2} h(x, y)}{\partial y \partial x}\right)_{(x, y)} d x d y \\
& =\int_{0}^{x} \int_{0}^{y} h(x, y) 2 \omega_{f} d x d y=2 \omega_{f} H_{x y}(x, y)
\end{aligned}
$$


$\mathrm{H}_{x y}$ is the primitive of $h(x, y)$ respect to the variables $x$ and $y$ and is defined as:

$$
H_{x y}(x, y)=f(0,0) x y+\left(\frac{y x^{2}}{2}\right) \theta_{x}+\left(\frac{x y^{2}}{2}\right) \theta_{y}+\left(\frac{y^{2} x^{2}}{4}\right) \omega_{f}
$$

The ICF of the bivariate linear function $h(x, y)$ is thus calculated as:

$$
\Delta E(x, y)=E_{0} / E_{I N}=f(0,0) x y / H_{x y}(x, y)
$$

\section{Results}

\subsection{The main evidence}

As anticipated in the introduction section, the research question of this paper wants to elucidate the significance (the implication, and the true meaning and nature) of the visually perceptible third dimension of the classic-curvature and the intensity-curvature functional images. The visually perceptible third dimension was initially reported ${ }^{[17]}$ pointing to the advantage of highlighting the tumor contour line. This paper reports on the implication of the visually perceptible third dimension of the classic-curvature and intensity-curvature images. The implication is to highlight the parts of the tumor mass where the fluids (more specifically, the contrast agent) tend to accumulate and in relationship to it, the following observations were made on the basis of the images shown in Figures 1-9, and 12.

(1) The contrast enhanced T1-weighted MRI shows the visually perceptible third dimension with a degree of visibility which is comparable to the one seen in the T2-weighted MRI (see Figures 3b, 3c, 6b, 6c, 7b, 7c versus Figures $1 b, 1 c$ and $2 b, 2 c$ ).

(2) The visually perceptible third dimension is more accentuated in the proximity of the regions where the contrast agent is accumulated, thus suggesting that it is determined by the fluids located in the human brain tumor (see Figures 6b, 6c and 7b, 7c versus Figures $9 \mathrm{~b}, 9 \mathrm{c}$, and also Figure 12).

(3) The T1-weighted MRI shows the visually perceptible third dimension with a degree of visibility which is less accentuated than the visibility obtained with the T2-weighted MRI and the contrast enhanced T1-MRI (see Figures 9b, 9c versus Figures 1b, 1c, 2b, 2c, 3b, $3 c, 6 b, 6 c$ and $7 b, 7 c)$.

(4) With the FLAIR pulse sequence, both classiccurvature and intensity-curvature functional show the visually perceptible third dimension (see Figures $8 \mathrm{~b}$, $8 \mathrm{c})$.

(5) In the T2-weighted MRI the classic-curvature shows clearly the visually perceptible third dimension (see Figures 1b, 1c, 2b, 2c, 4b, 4c and 5b, 5c).
(6) The white/bright regions of the human brain visible in the T2-weighted MRI are displayed with the visually perceptible third dimension in both classic-curvature and intensity-curvature images (see Figures 1b, 1c, 4b, $4 c$ and $5 b, 5 c)$. This phenomenon suggests that the visually perceptible third dimension is related to the fluids in the human brain.

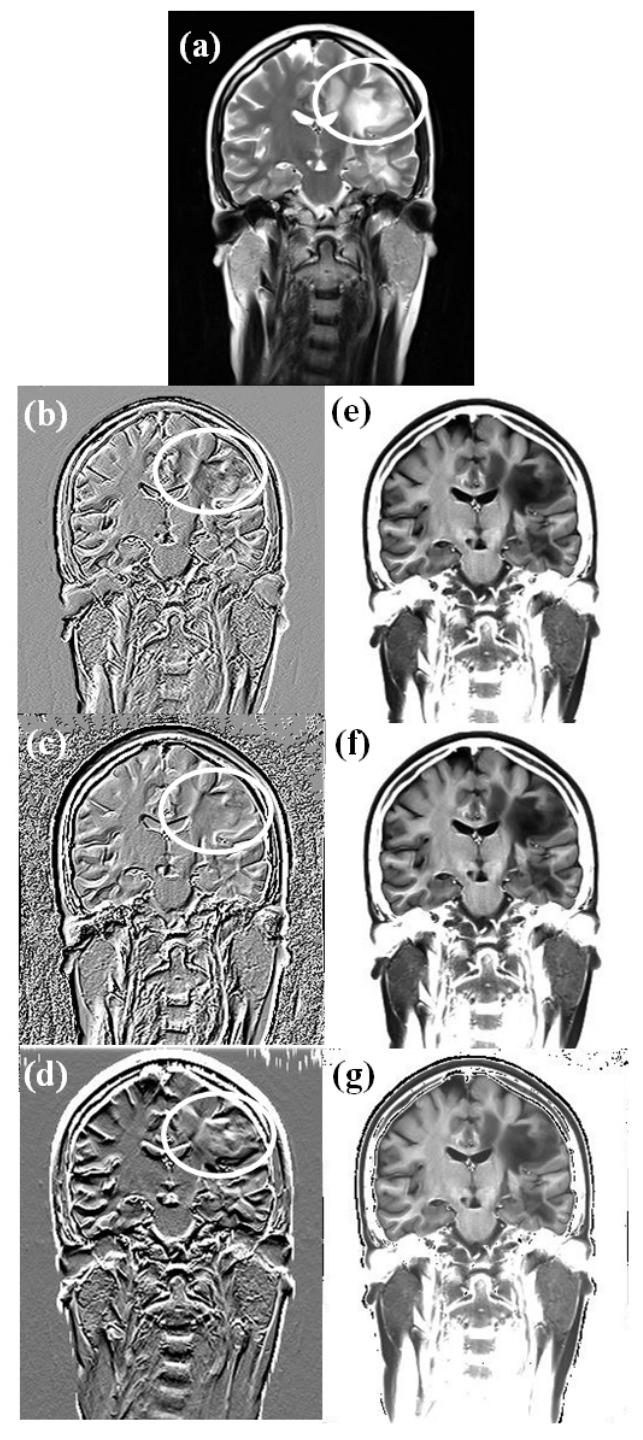

Figure 1. Sagittal T2-weighted MRI showing brain metastases in (a), classic-curvature image calculated with the bivariate cubic polynomial in (b), and in (c) the intensity-curvature functional image calculated with the bivariate linear model function. The image in (d) is the high pass filtered MRI and the image in (g) was obtained convolving the image in (a) with the image in (d). Similarly, the classic-curvature image (b) and the intensity-curvature functional image (c) were convolved with the MRI shown in (a) and the result of the convolution is shown in (e) and in (f) respectively. The MRI in (a) is the same as the one shown in Figure $4 \mathrm{a}$ in previous work. ${ }^{[14]}$ 
Generally, on the basis of the aforementioned observations the visually perceptible third dimension is more accentuated in the following MRI imaging modalities: contrast enhanced T1-weighted MRI, T2-weighted MRI. And, the aforementioned MRI modalities are all capable to increase the MRI signal of the fluids. In the specific cases presented in this manuscript, the fluids tend to accumulate in the tumor regions and such accumulation determines as to why the visually perceptible third dimension is more accentuated. In the specific case of the FLAIR imaging modality, which tends to suppress the MRI signal of the cerebrospinal fluid (CSF), the most immediate observation is that the visually perceptible third dimension is more accentuated at the borderline of the human brain ventricles (where the CSF resides). Nevertheless, the aforementioned observations provide with an explanation on the implication of the visually perceptible third dimension which is that of highlighting the accumulation of fluids in the human brain.

The investigation reported in Figures 1-9 makes evidence on the meaning and the nature of the visually perceptible third dimension of the classic-curvature and the intensitycurvature functional images, which is suggested to be the high pass filtered MRI signal. To obtain this finding the following procedure was undertaken. The high pass filtered MRI was calculated and is shown in Figures 1d, 2d, 3d, 4d, 5d, 6d, 7d, 8d and 9d. The high pass filtered MRI was convolved to the MRI so to obtain the images shown in Figures $1 \mathrm{~g}, 2 \mathrm{~g}, 3 \mathrm{~g}, 4 \mathrm{~g}, 5 \mathrm{~g}, 6 \mathrm{~g}, 7 \mathrm{~g}, 8 \mathrm{~g}$ and $9 \mathrm{~g}$.

Figures 1-9 show the classic-curvature and the intensitycurvature images in (b) and in (c) respectively, which were treated as if they were filter masks, and so they were convolved with the MRI. The images obtained in (e) and (f) of each figure (1-9) are the result of the convolution of the classic-curvature and the intensity-curvature images with the MRI image respectively, and they are quite similar to the images shown in $(\mathrm{g})$.

Although the paradigm used does not suffice to provide with a mathematical proof to the aforementioned assertion, it does provide with empirical evidence. The empirical evidence is made through two relevant and important results. One result is the striking similarity between the high pass filtered MRI images and both the classic-curvature and the intensity-curvature functional images. And the aforementioned result makes the point that the two aforementioned intensity-curvature measurement approaches (CC and ICF) appear as high pass filters. More importantly, the second result consist of the striking similarity of the images obtained through the convolution of the MRI with the three filters, which makes the point that both $\mathrm{CC}$ and ICF are suggested to be high pass filters.
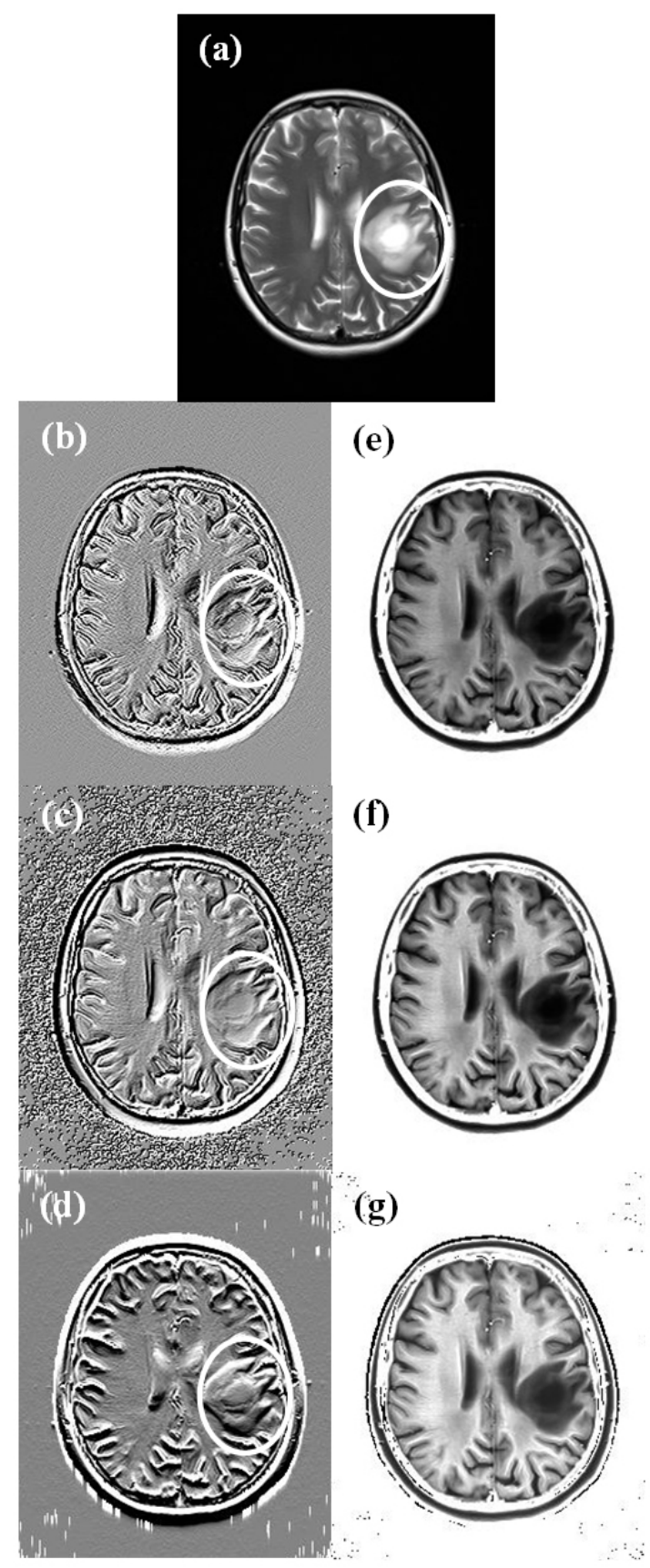

Figure 2. T2-weighted pulse sequence MRI in the transverse plane showing brain metastases in (a). The classic-curvature image was calculated with the bivariate cubic polynomial and is shown in (b), whereas (c) shows the intensity-curvature functional image calculated with the bivariate linear model function. The classic-curvature and the intensity-curvature functional images were convolved with the MRI shown in (a) and the result is shown in (e) and in (f). The image in (d) is the high pass filtered MRI which was used to convolve the MRI in (a) so to obtain the image shown in $(\mathrm{g})$. The MRI in (a) is the same as the one shown in Figure $3 \mathrm{a}$ in previous work. ${ }^{[15]}$ 


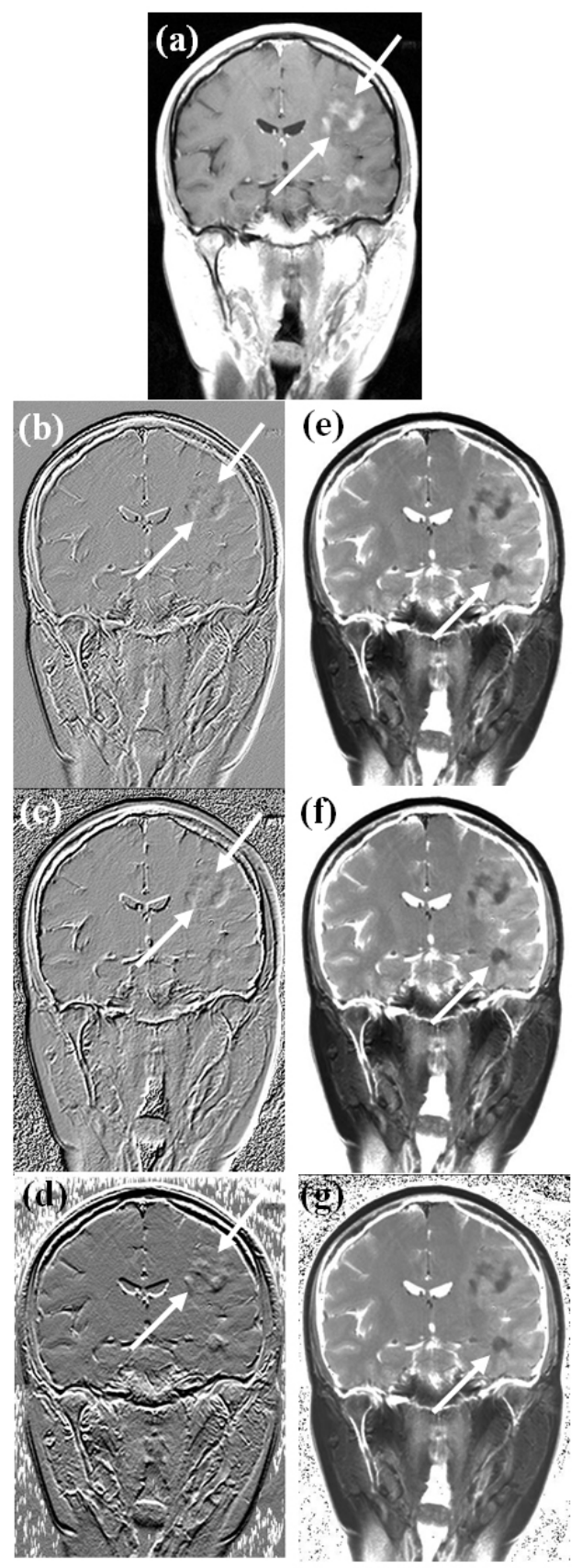

Figure 3. A contrast enhanced T1-weigthed MRI showing brain metastases in (a). The images in (b) and in (c) show the classic-curvature and the intensity-curvature functional respectively, which were calculated with the bivariate cubic polynomial (b) and the bivariate linear model function (c). The image in (d) is the high pass filtered MRI, and the images in (b) and in (c) were treated as if they were high pass filters of the MRI shown in (a). Therefore the images in (e), (f) and (g) are all resulting from processing the image in (a) through the convolution with the high pass filters shown in (b), (c) and (d) respectively.
Figure 1 shows in (e), in (f) and in (g) the filtered images. The following observations can be made: (1) the distinction between grey and white matter of the brain is clear, and (2) the tumor mass is clearly highlighted however not elevated respect to the image plane as it is in (b), in (c) and in (d), which are the classic-curvature, the intensity-curvature functional and the high pass filtered MRI respectively. Both (b) and (c) show the visually perceptible third dimension mostly visible at the location of the metastases as shown by the region of the brain inside the white ellipses. Magnified details of the visually perceptible third dimension are seen inside the ellipse in (b) versus those shown inside the ellipse in (c). It is important to report that the images in Figures 1a, 2a, 3a, 4a, 5a, 6a, 7a, 8a, 9a and 10a.1, were contrastbrightness enhanced in order to increase the visibility of the tumor regions.

Observations similar to those made while commenting Figure 1 can be made on the basis of Figures $2 \mathrm{e}, 2 \mathrm{f}$ and $2 \mathrm{~g}$, with the additional commentary that the three images do not show the same contrast-brightness enhancement. The contrastbrightness enhancement was not set to be the same with the objective to have a clear visualization of each image. The cystic part of the tumor is more discernible in (f) and in (g) than it is in (e). The classic-curvature, the intensity-curvature functional, and the high pass filtered MRI images in (b), in (c) and in (d) respectively, show the visually perceptible third dimension at the location of the tumor mass (see inside the white ellipses). Looking inside the ellipses, there is a visible increase of the level of details in (b) versus (c). The same paradigm used to obtain the filtered images shown in Figure 1 was used in the case shown in Figure 2.

Figures $3 \mathrm{e}, 3 \mathrm{f}$ and $3 \mathrm{~g}$ show a detail of the tumor (see arrows in (e), (f) and (g)), which is also visible in the MRI image (a), in the classic-curvature image (b) and in the intensitycurvature functional image (c). The detail consist of the part of the tumor mass which appears disjointed from the main one indicated by the two arrows in (a), (b) and (c). The level of details is slightly finer in (c) than it is in (b). The two aforementioned pictures provide evidence in support to the hypothesis that the visually perceptible third dimension appears at the location of major accumulation of fluids, which in the specific case is the contrast agent (see the tumor mass indicated by the arrows in (b) and in (c)). Overall the figures in (e), (f) and (g) present an enhanced grey level scale which makes it possible to observe the tumor masses with magnified details and highlight the tumor through a grey level which makes the contrast higher versus the rest of the brain. 


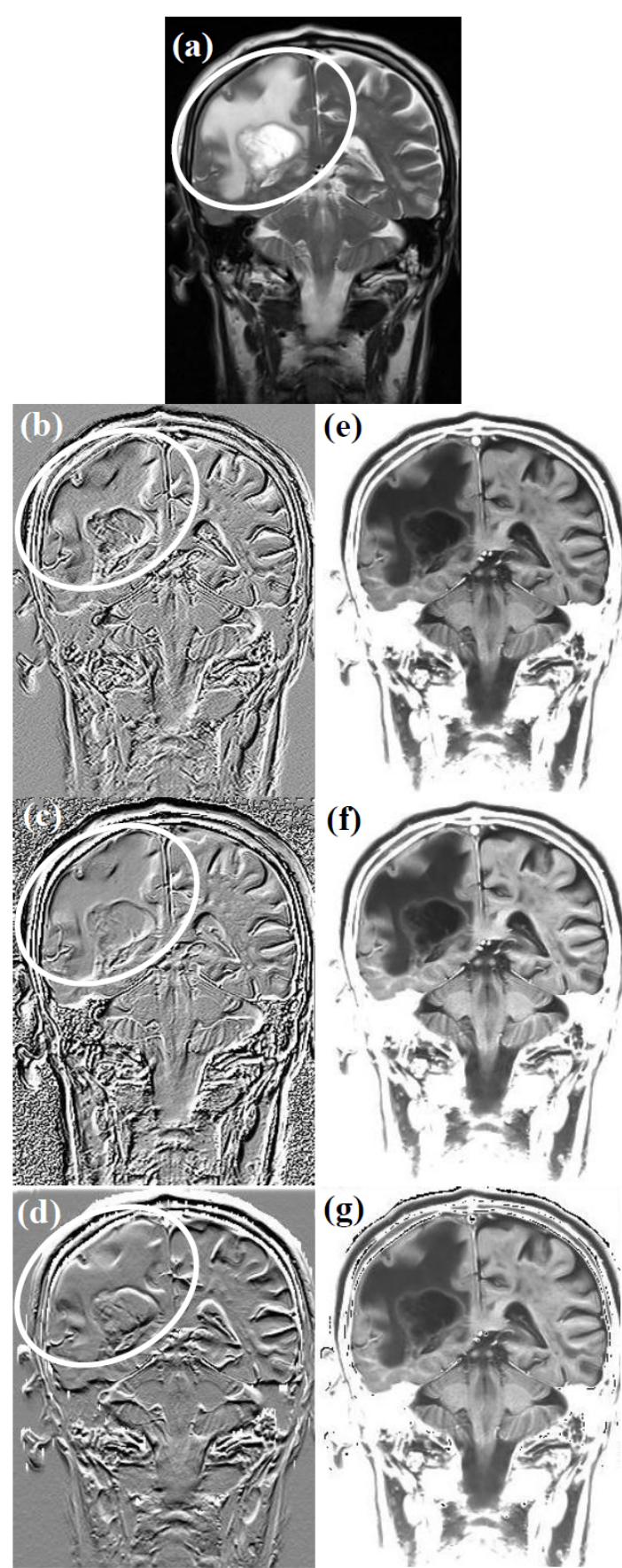

Figure 4. A coronal T2-weighted MRI showing a glioblastoma multiforme is displayed in (a), and the classic-curvature image which was calculated with the bivariate cubic polynomial is shown in (b). The image in (c) is the intensity-curvature functional image of (a) and it was calculated with the bivariate linear model function. The image in (d) is the high pass filtered MRI. The images in (b), (c) and in (d) were convolved with the MRI shown in (a), so to obtain the images shown in (e), (f) and in (g) respectively.

In Figure 4, it is visible the similarity between the classiccurvature image (b), the intensity-curvature functional image (c) and the high pass filtered MRI (d). The MRI shown in (a) was convolved with the images in (b), (c) and in (d), which were therefore used as filter masks, so to obtain the images in (e), (f) and in (g) respectively. Because of the similarity between the images in (b), (c) and in (d), and because of the aforementioned convolution (i.e. the process of convolving the MRI in (a) with the images shown in (b), (c) and in (d) also the filtered images shown in (e), (f) and in (g) show substantial degree of similarity among each other. The edema is shown in dark in the filtered images (e), (f) and $(\mathrm{g})$, and is well distinct from the rest of the human cortex. Additionally, the darkest color in (e), (f) and (g) is shown by the cystic central part of the tumor mass, as well by the right ventricle. Both of (b) and (c) show the visually perceptible third dimension at the location of the metastases as shown inside the region of the brain tumor enclosed into the white ellipses. The level of details of the tumor region is finer in (b) than it is in (c). The characteristic of the filtered images is to highlight the structure of the tumor, which is made of three major constituent parts: (1) the cystic part placed at the center of the tumor mass, (2) the borderline of the cystic part of the tumor, which is highlighted through a lighter grey color, and (3) the rest of the tumor mass which goes beyond the borderline of the cystic part and is highlighted in dark grey. The images in (e), (f) and in (g) show remarkable similarity with the MRI seen in (a), for what pertains to the information content related to the tumor.

As visible in Figures 1-4, the visually perceptible third dimension appears also in the high pass filtered MRI in (d). And, the image in (d) shows a more accentuated visually perceptible third dimension when compared to the classiccurvature and intensity-curvature functional images. The aforementioned aspect becomes even more evident when looking at the Figures $5 b, 5 c$ and $5 d$. Notwithstanding the aforementioned feature, the result of the convolution is quite similar in the three cases presented in (e), (f) and in (g) of the figures, and this aspect supports the evidence herein provided that both of the classic-curvature and the intensity-curvature functional images are suggested to be the high pass filtered MRI signal.

In Figure 5, both (b) and (c) show the visually perceptible third dimension at the location of the tumor as shown by the region of the brain inside the white ellipses. Also in this case, the image in (b) shows magnified details compared to those details visible in the image in (c). Likewise observable in Figures 1-9, the visually perceptible third dimension appears also in the high pass filtered MRI seen in Figure 5d, where is more accentuated (see Figure 5d versus Figure 5b and Figure $5 c)$. 

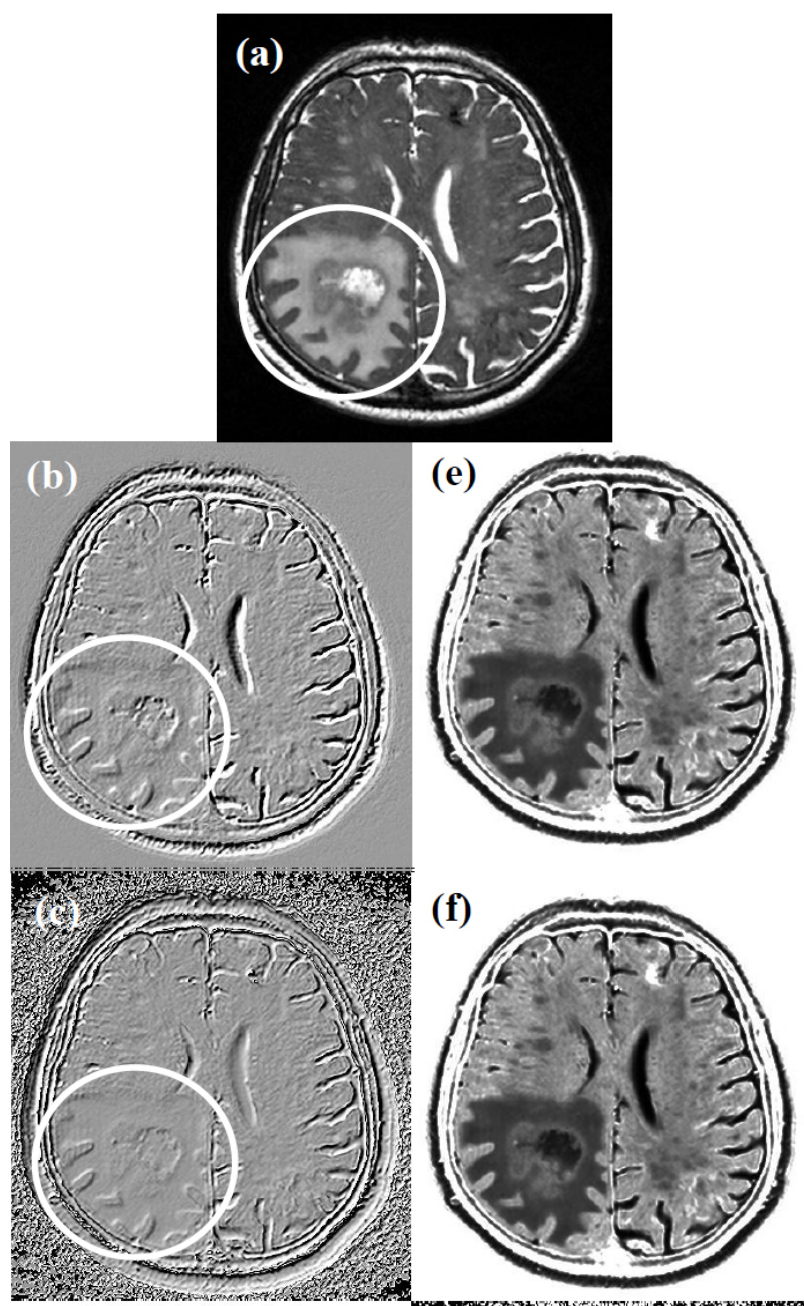

(f)
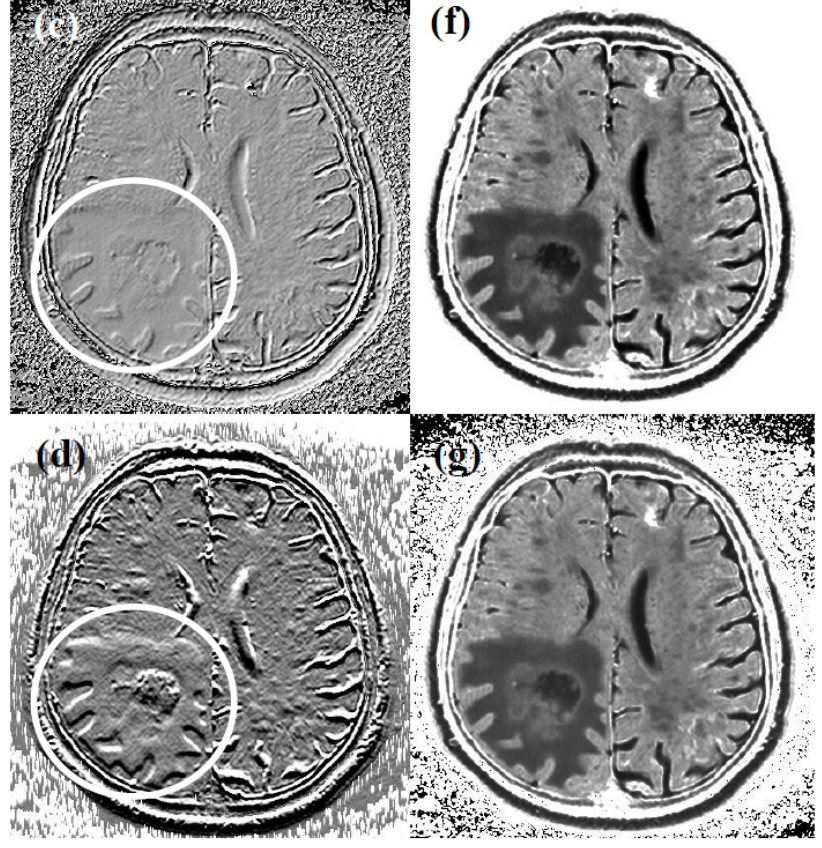

Figure 5. A T2-weighted MRI in the transverse plane showing the glioblastoma multiforme is shown in (a). The classic-curvature image of (a) is shown in the image in (b) and was calculated with the bivariate cubic polynomial. The image in (c) shows the intensity-curvature functional of (a) and was calculated with the bivariate linear model function. The image in (d) is the high pass filtered MRI.The images shown in (e), (f) and in (g) were obtained convolving the MRI in (a) with the images shown in (b), (c) and in (d) respectively.

Observations made on the basis of Figures 6e, $6 f$ and $6 \mathrm{~g}$ reveal that: (1) the tumor mass is well distinct in terms of grey levels from the rest of the human brain cortex, (2) the tumor mass is well represented in its structure through three main grey levels, (3) the cystic part of the tumor appears with the darkest grey and is related to the images in (b), (c) and (d) by means of the highest elevation observable through the visually perceptible third dimension. As seen in Figure 3 (which also shows a contrast enhanced T1-weigthed MRI) the major accumulation of contrast agent is at the location of the tumor (see inside the white ellipses in (b) and in (c)). And at the aforementioned location the visually perceptible third dimension perpendicular to the image plane has its major appearance. It is worth noting that filtering enhances the contrast-brightness and this is visible in the filtered images shown in (e), (f) and in (g), when compared to the MRI (a). The aforementioned enhancement provides the imaging of the tumor with particular emphasis and information content about the anatomical structure. It is due to acknowledge that, although arbitrary in the extent, the contrast-brightness enhancement is essential to the visibility of the filtered images reported in this paper.

As already seen in Figures 1, 2 and 3, the analogy between the images shown in Figure 6 in (b), in (c) and in (d) and the convolved images shown in (e), in (f) and in (g), consists of the localization of the cystic part of the tumor. The location of the cystic part of the tumor is elevated along the visually perceptible third dimension in the classic-curvature image (b), and in the intensity-curvature functional image (c) and in the high pass filtered MRI (d). And, at the same time, the cystic part of the tumor is shown as the darkest region in the convolved images obtained applying the three masks to the MRI. Particularly interesting is the capability to visualize the entire region of the edema of the images in (e) and in (f), which are derived from the classic-curvature image in (b) and the intensity-curvature functional image in (c) respectively. The whole region of the edema is visible in (a) (see inside the white circle) and is emphasized in the filtered images (see grey color in the lower right region of the edema in (e) and in (f)).

Also, in Figure 6, the main feature of the mask images: (b), (c) and (d); is correlated with the imaging and the accumulation of the contrast agent in proximity and inside the tumor mass. The result of filtering is similar in all of the Figures 6e, of and $6 \mathrm{~g}$ where the tumor is imaged in its structure showing a well discernible aspect which makes it different from the rest of the human brain cortex. In Figure 6, likewise shown already in Figures 3 and 4, the filtered images in (e), (f) and (g) appear illuminated in a similar fashion seen already in previous research through the signal resilient to interpolation. ${ }^{[21]}$ This aspect will become more apparent in Figures 7, 8 and 9. 

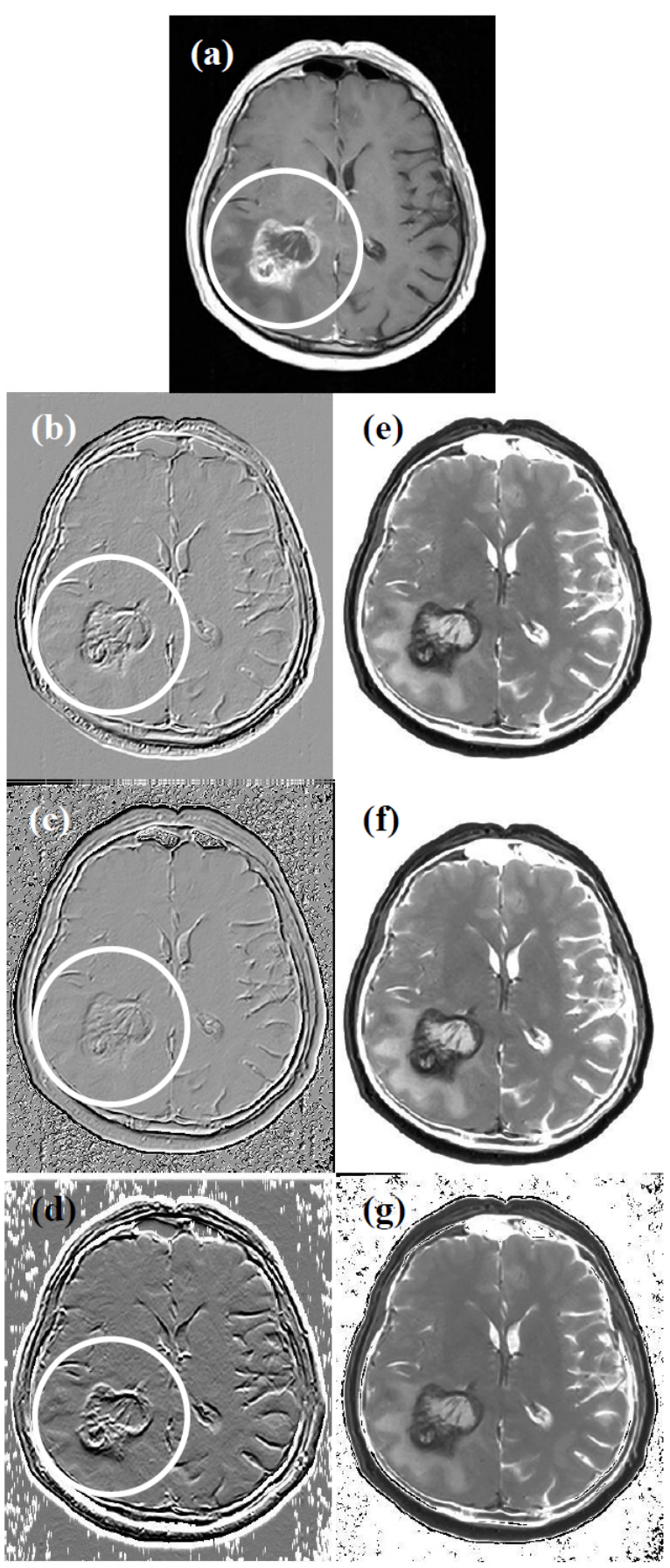

Figure 6. A glioblastoma multiforme shown with a contrast enhanced T1-weighted MRI in the transverse plane (a). The images in (b) and in (c) show the classic-curvature and the intensity-curvature functional images calculated with the bivariate cubic polynomial and the bivariate linear model function respectively. The image in (d) is the high pass filtered MRI. As the previous cases shown in Figures 1 through 5 the images in (b), in (c) and in (d) were convolved with the MRI shown in (a), so to obtain the filtered images shown in (e), (f) and in (g).
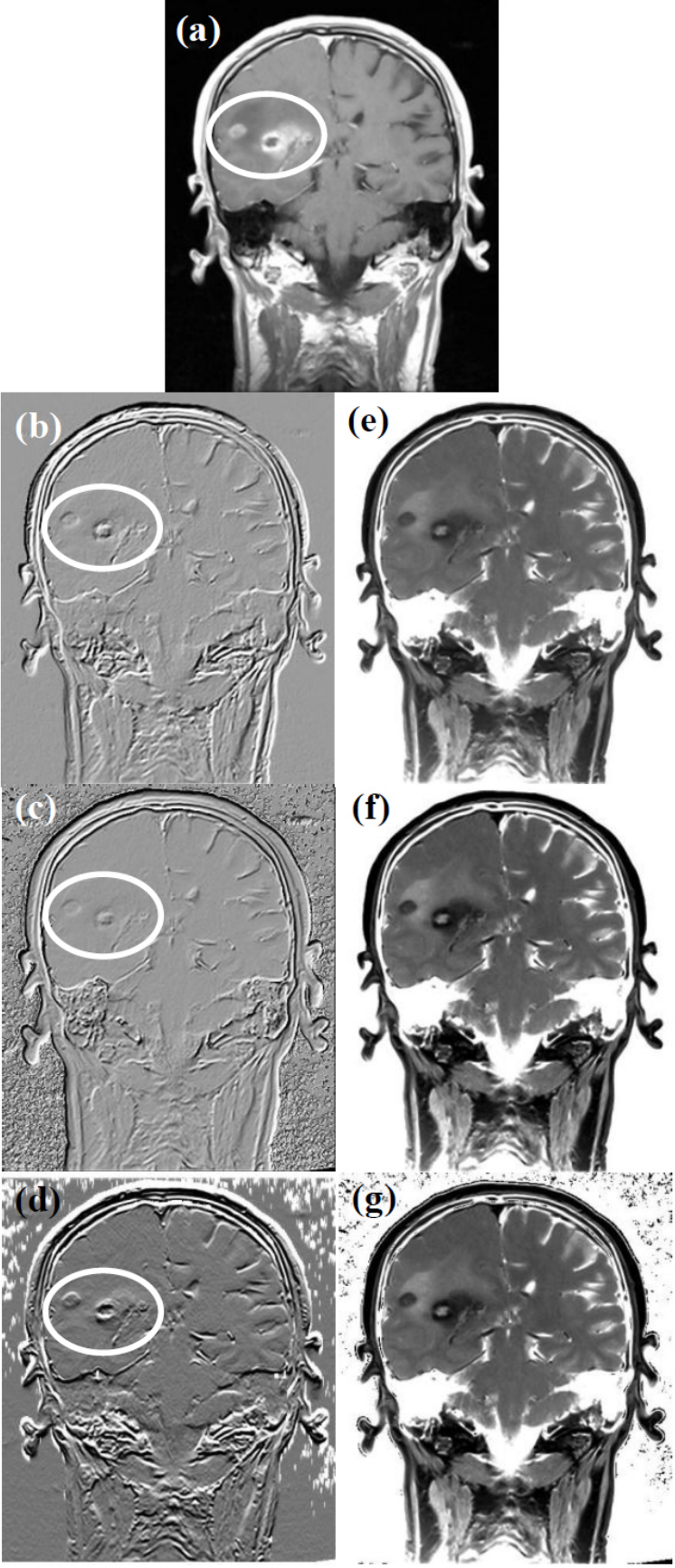

Figure 7. Glioblastoma multiforme shown through a contrast enhanced T1-weighted MRI in the transverse plane (a). The classic-curvature image (calculated with the bivariate cubic polynomial) and the intensity-curvature functional image (calculated with the bivariate linear model function) are shown in (b) and in (c) respectively. The image in (d) is the high pass filtered MRI. The images in (e), (f) and $(\mathrm{g})$ were obtained convolving the MRI shown in (a) with the classic-curvature image (b), the intensity-curvature functional image (c) and the high pass filtered MRI (d), respectively. 


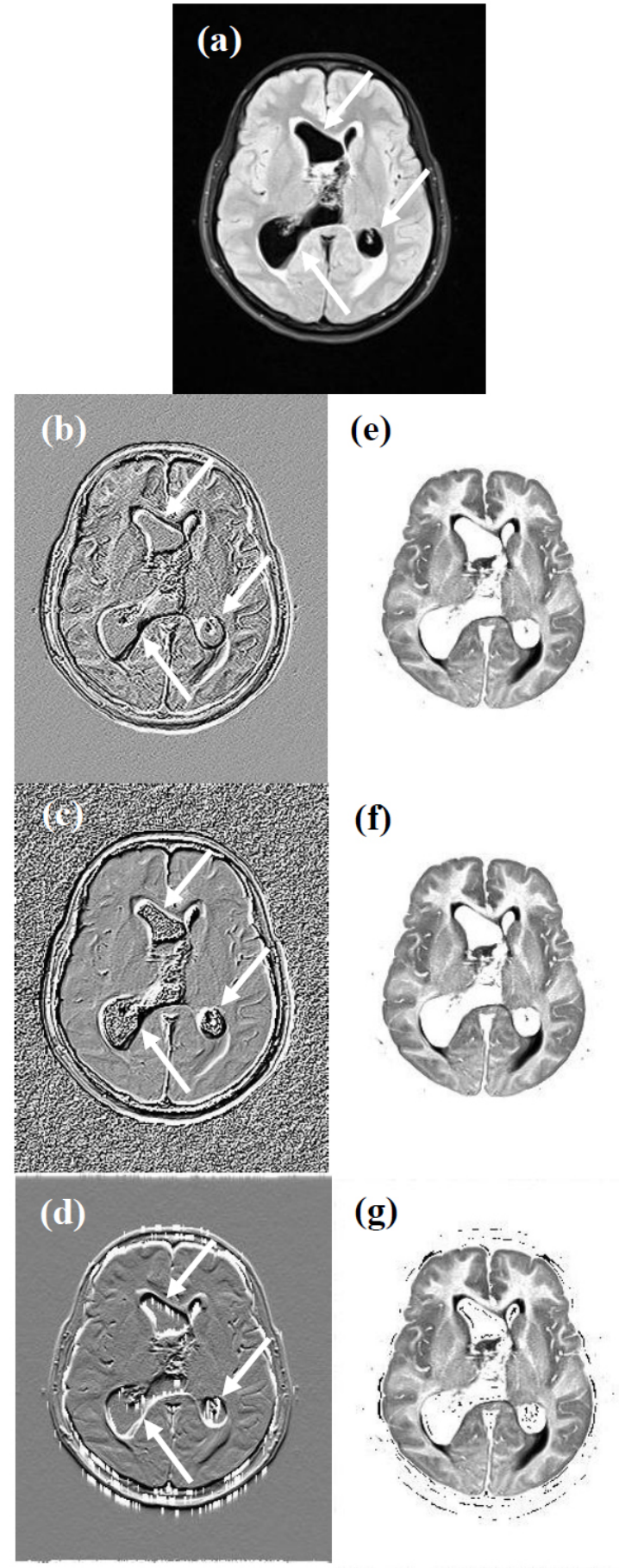

Figure 8. The intra-ventricular brain tumor is shown in (a) through the FLAIR pulse sequence image in the transverse plane. The classic-curvature image (b) and the intensity-curvature functional image (c) were calculated with a bivariate cubic polynomial and the bivariate linear model function respectively. The image in (d) is the high pass filtered MRI. The two main characteristics of the filtered images shown in (e), (f) and in (g) are: (1) the capability to distinguish between the tumor and the CSF, and (2) the capability to distinguish between grey and white matter.

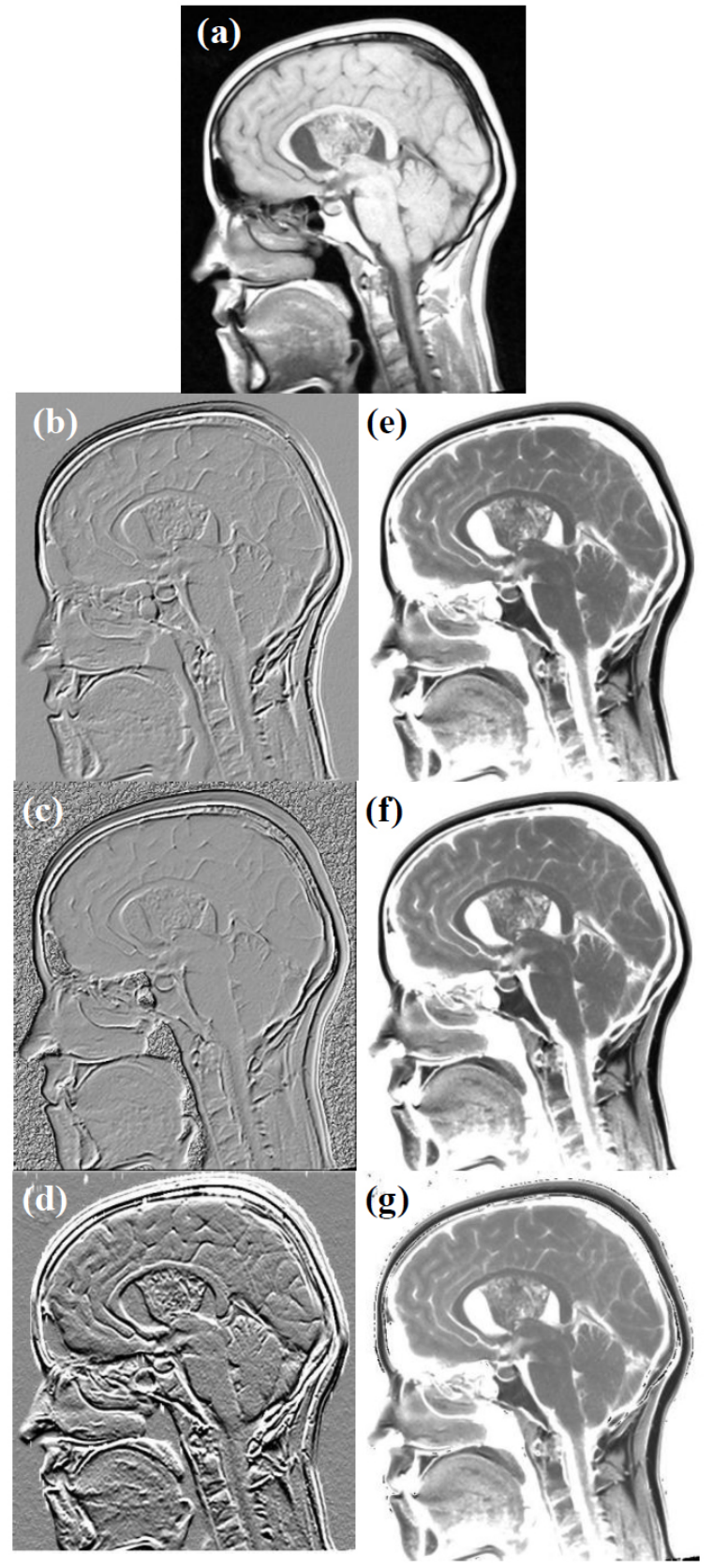

Figure 9. An intra-ventricular brain tumor is shown through a sagittal T1-weighted MRI in (a). The images in (b) and in (c) are the classic-curvature and the intensity-curvature functional images calculated with a bivariate cubic polynomial and the bivariate linear model function respectively. Magnified details are observable in (d) versus (b) and (c). The lack of contrast agent acts in favor to the reduction of the visually perceptible third dimension in both (b) and (c), but not in the high pass filtered MRI shown in (d). The characteristics of the filtered images (e), (f) and (g) are: (1) a contrast-brightness effect which makes it possible to highlight the features of the intra-ventricular tumor; and (2) the imaging of the sulci and gyri, which makes it possible to observe the anatomy the details of the brain cortex. 
The tumor structure, centered in the two circular edemas, is well visible in the convolved images shown in Figure 7e, 7f, and $7 \mathrm{~g}$ along with the surrounding regions where the contrast agent tends to accumulate. The dark grey levels highlight the rest of the tumor mass. The convolved images, obtained using the classic-curvature image and the intensity-curvature functional image as filter masks, provide diagnostic settings with complementary and/or additional information to the MRI shown in (a). The accumulation of the contrast agent at the location of the tumor (see inside the white ellipses in (b) and in (c)) makes additional evidence that the visually perceptible third dimension is related to the presence of fluids in the human brain.

The images in Figure $8 \mathrm{~b}$ and $8 \mathrm{c}$ show that the visually perceptible third dimension is more accentuated inside the ventricles where the CSF resides. However, the CSF is located at a lower elevation (see arrows in (b) and in (c)) when compared with the rest of the human cortex. The main characteristic of Figures $8 \mathrm{~b}, 8 \mathrm{c}$ and $8 \mathrm{~d}$ is that the visually perceptible third dimension distinguishes the ventricles of the brain from the rest of the cortex. This distinction does appear in Figures 8e, $8 \mathrm{f}$, and $8 \mathrm{~g}$, as it is visible in the white regions of the three convolved images. The region inside the ventricles, which is CSF and tumor mass, appears in white. In the case presented in Figure 8, is not possible to discern complementary and/or additional information from the images in (e), in ( $f$ ) and in (g). However, it is possible to have a contribution through the analysis of the images in (b), in (c) and in (d) because of the possibility to distinguish between the tumor mass and the CSF. The tumor structure is well localized in all of the images in (e), (f) and (g) at the center of the ventricles, which appear deeply affected in their anatomical structure.

The images in Figure $9 \mathrm{~b}$ and 9c show less evident visually perceptible third dimension than the one shown in the high pass filtered MRI of Figure 9d. Additionally, the convolved images in (e), (f) and in (g), appear illuminated and so they present a grey scale magnified towards the high values of pixel intensity, which makes it easier for the observer to visualize the details of the tumor structure. Indeed, Figures $9 \mathrm{e}, 9 \mathrm{f}$ and $9 \mathrm{~g}$ show that the tumor mass is clearly discernible inside the ventricle.

\subsection{Additional evidence}

This section reports evidence that the pulse sequence called FLAIR $^{[19]}$ can benefit of the filtering performed through the convolution of the MRI with the intensity-curvature functional image. Indeed, an observation is possible through the analysis of Figure 10, in (a.1) (FLAIR MRI), in (b) (ICF image of the FLAIR MRI calculated with the bivariate linear function), and in (c) (FLAIR MRI filtered with the ICF image). The observation is that the level of details seen in the filtered MRI (see Figure 10c inside the black ellipse) is enhanced so to show features of the tumor not observable in the FLAIR MRI (a). The contrast-brightness enhancement in (a.2) is provided to make sure that the details seen in (c) cannot be seen through a simple contrast-brightness enhancement of (a.1). Thus, the information provided in (a.2) supports the usefulness of the filtering shown in (c). When filtering FLAIR data, the aforementioned behavior of the intensity-curvature functional image has been observed in four subjects out of eight and is a novelty in the literature.

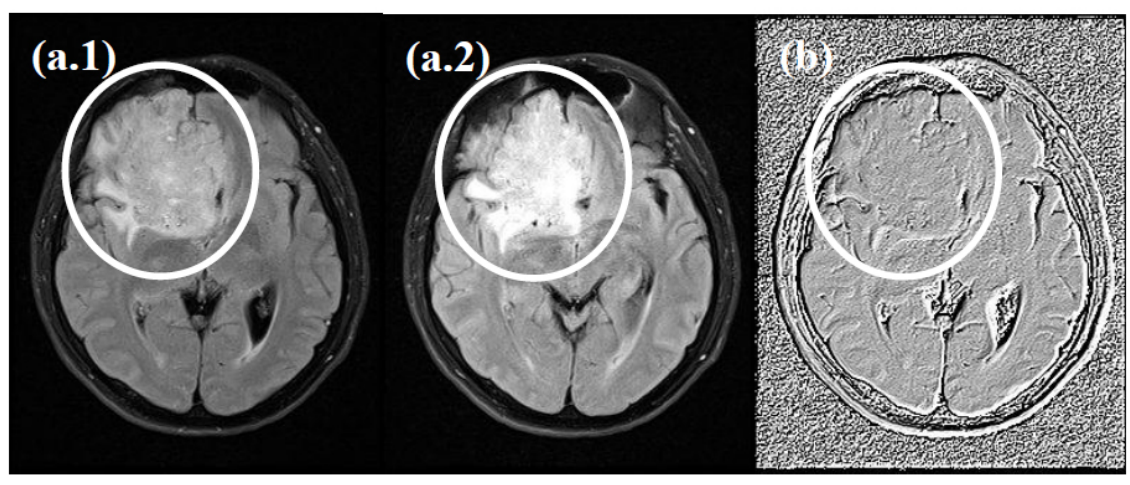

(c)

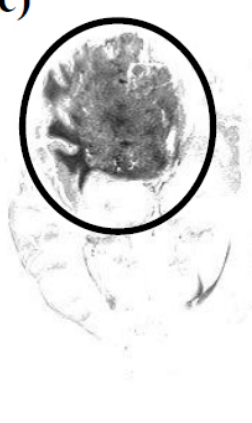

Figure 10. In (a.1) is shown the collected FLAIR MRI with emphasis on the tumor region enclosed inside the white ellipse. In (a.2) the FLAIR MRI is contrast-brightness enhanced. In (b) is the intensity-curvature functional (ICF) image of the FLAIR MRI, which is calculated with the bivariate linear function. The convolution of the FLAIR image in (a.1) with the ICF in (b) is shown in the filtered FLAIR MRI in (c). Note that the level of details of the tumor in (c) is more accentuated than the level of details shown in (a.1) and in (a.2). See the regions inside the white ellipse in (a.1) and in (a.2) versus the regions inside the black ellipse in (c). 


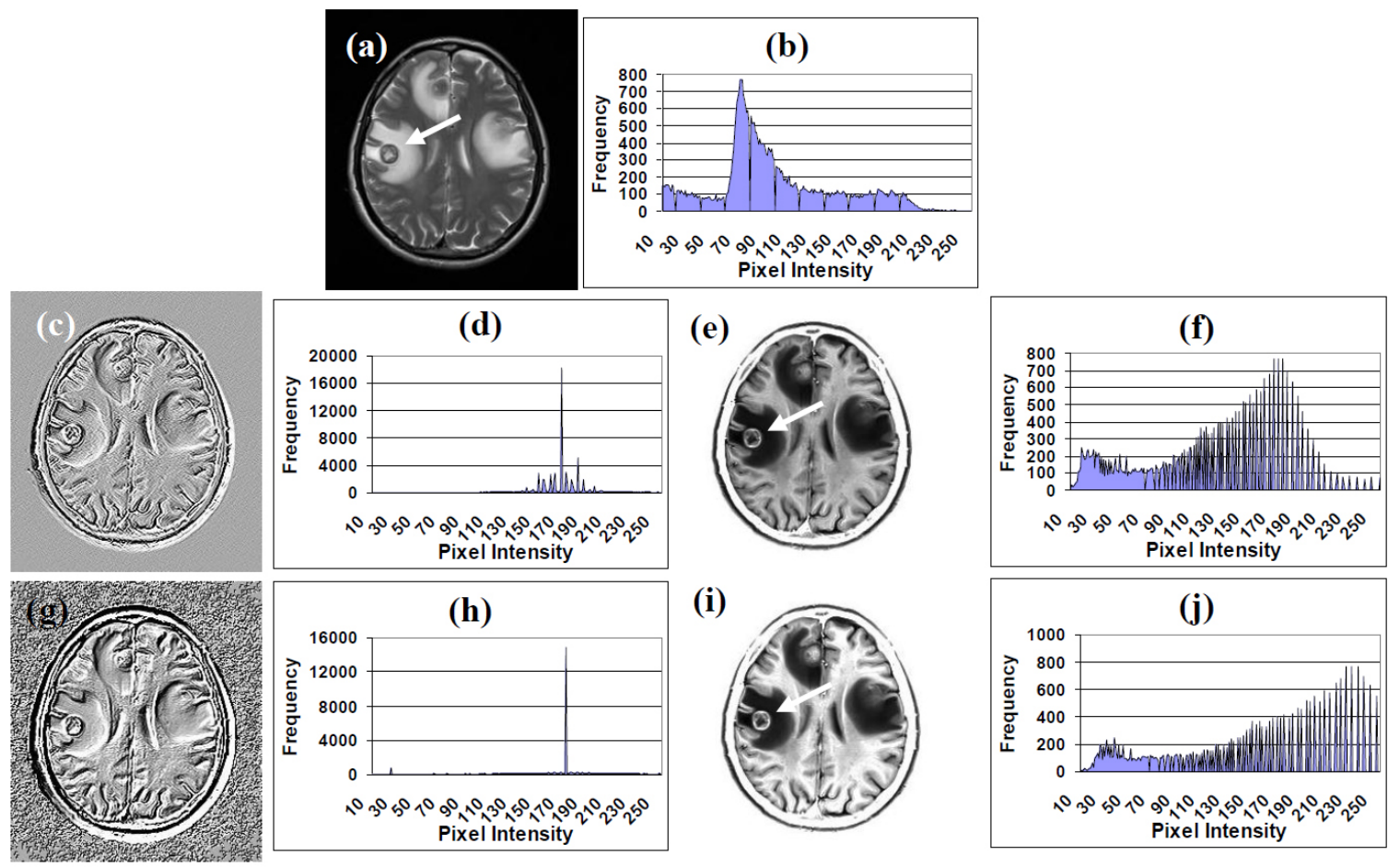

Figure 11. The T2-weighted MRI of brain metastases is shown in (a), the CC image is shown in (c), the ICF image is shown in (g). The model functions are: the bivariate cubic polynomial (c), and the bivariate linear function (g). The MRI shown in (a) is convolved with the CC image shown in (c) and the ICF image shown in (g), so to obtain the filtered images in (e) and (i) respectively. In (b), (d), (f), (h) and (j) are the histograms of the images shown in (a), (c), (e), (g) and (i) respectively.

The CC and the ICF images were reported earlier as medical intensity-curvature maps while elucidating their characteristics and pointing out to the capability of such images to provide the diagnostic setting with the property of highlighting the tumor contour line. ${ }^{[14,17]}$ Figure 11 reports an additional example of the aforementioned capability, specifically, when the imaging modality is the T2-weighted MRI (see Figure $11 \mathrm{c}$, which is the CC image; and Figure $11 \mathrm{~g}$, which is the ICF image). Moreover, another example of the meaning and the nature of the CC and the ICF images is presented in Figure 11. The additional evidence here provided, reinforces on the fact that the CC and the ICF images are filter masks which when convolved with the MRI make it possible to further the filtering of the MRI (see Figures 11e and 11i). Thus, the assessment of the true meaning and the nature of the $\mathrm{CC}$ and the ICF images is the justification of their calculation.

Additionally, as shown in Figure 10, the justification of the calculation of the ICF images is provided by the property of filtering out the MRI image (the FLAIR imaging modality in the specific case) and to highlight features of the tumor which are not readily observable.

The necessity of the contrast-brightness enhancements of the filtered images is related to the grey levels of the images and such enhancement allows seeing the image details in its full extent. The quality of the resulting filtered images is fair and tailored to the intended use. The application of the ICF and the CC images as filter masks make it possible to see the intensity-curvature based filtered MRI for the first time in the literature in such extensive, comprehensive and detailed manner. However, in recent works, ${ }^{[14]}$ there is mention to similar results obtained through the use of the signal resilient to interpolation (which is shown to be similar to a filtered MRI).

\section{Discussion}

\subsection{The literature in reference to the research effort}

Because of the characteristics of the $\mathrm{CC}$ and the ICF images it is relevant to mention literature related to the localization of the tumor. The localization can be achieved through MRI post-processing techniques based on segmentation which rely on the enhancement of the tumor contour line delineation obtained with contrast enhanced T1-weighted MRI. ${ }^{[22]}$ In our research, the tumor contour line can be delineated through the use of both the intensity-curvature functional and the classic-curvature images. Moreover, we have observed an accentuation of the visually perceptible third dimension in the proximity of the accumulation of the contrast agent in the area of the human brain affected by the tumor mass. The 
aforementioned phenomenon is the implication of the visually perceptible third dimension of the $\mathrm{CC}$ and the ICF images.

As far as regards filtering, wavelet based filtering is the state of the art approach. ${ }^{[23]}$ The literature reported in this section of the paper is related to filtering of human brain MRI data. The noise in MRI is white Gaussian and additive in both of the real and imaginary parts of the $k$-space and since the Fourier transformation (FT) is a liner process, the noise remains Gaussian after the FT transformation yields the reconstructed MRI in the image space and the noise becomes Rician when the absolute value is taken into account. ${ }^{[2,25]}$ The noise in MRI, or more specifically, the bias field, is also known as the intensity inhomogeneity and it is the nonuniform pixel intensity value of the same tissue in the image space. ${ }^{[25]}$ The main assumption in spatial filtering in MRI is that the intensity inhomogeneity can be removed through low pass filtering, however this assumption does not hold true in MRI scanning of the human brain because of the size of the head. ${ }^{[26]}$ On the basis of the aforementioned assumption, the noise, which manifests itself as a smooth intensity variation across the image, ${ }^{[26]}$ resides in the low frequency domain. Therefore, in order to remove the noise from the MRI, it is common to separate the MRI image from the extracted intensity inhomogeneity of the image. ${ }^{[25]}$ The aforementioned process can be implemented through spatial filtering which can be usually performed with two types of operations. One is low pass filtering of the MRI, which can be done through median filters, or through mean filters, or on the basis of the multiplication in the Fourier domain. ${ }^{[26]}$ And, the other is homomorphic filtering which works on the basis of the low pass filtered image. ${ }^{[27,28]}$

Anisotropic diffusion filters of MRI images had been reported. ${ }^{[29]}$ The Gaussian filter of MRI images has been introduced ${ }^{[30]}$ but it shows the disadvantage of removing high frequency components. An optimized block-wise non-local means filter for 3D MRI volumes was proposed and was shown to be capable of noise removal and at the same time of keeping the integrity of the MRI images. ${ }^{[31]}$ Pre-smoothing with non-local means filter applied to MRI of Alzheimer's disease patients have also been reported. ${ }^{[32]}$ Filters applied to DTI-MRI assuming that the diffusion tensor is a suitable approximation of an anisotropic Gaussian filter function had been reported. ${ }^{[33]}$ Additionally, two methods were reported which filter the Rician noise added to the MRI through the generation of Gaussian noise in both real and imaginary parts of the image. ${ }^{[34]}$

In the works presented in this paper, filtering is also related to the removal of the low frequency components of the MRI

Published by Sciedu Press signal. Indeed, filtering is here performed through the CC and the ICF images which are treated as filter masks. The $\mathrm{CC}$ and the ICF images can build the visually perceptible third dimension which can highlight the tumor structures and anatomy of the human brain (see also Figures $5 \mathrm{e}$ and $5 \mathrm{f}$ in previous work ${ }^{[21]}$ as far as regards the ICF). The meaning and the nature of the visually perceptible third dimension in the CC and the ICF images is the capability to filter the MRI. And thus, MRI filtering is performed in this paper through the use of the CC and the ICF filter masks.

\subsection{The implication, the meaning and the nature of the $\mathrm{CC}$ and the ICF images}

The main challenge of the works reported in this paper is to assess the implication, the meaning and the nature of the visually perceptible third dimension observed in the classiccurvature and the intensity-curvature functional images calculated with the bivariate cubic polynomial and the bivariate linear function respectively.

One implication of the visually perceptible third dimension of 2D MRI images is to provide the MRI images with the contour line of the tumor. ${ }^{[17]}$ However, the main implication of the visually perceptible third dimension was not addressed so far in full, and as such, it is investigated in the present works. This paper reports that the visually perceptible third dimension seen in 2D tumor MRI images ${ }^{[18]}$ through the classic-curvature and the intensity-curvature functional images is consequential to the accumulation of fluids in the human brain (more specifically, the contrast agent and/or the fluids of the tumor). Additional results are presented in Figure 12 in support to the aforementioned statement.

Figure 12 addresses the implication of the visually perceptible third dimension at the location of the tumor mass in the $\mathrm{CC}$ images. The implication consists of the phenomenon of increased visibility of the visually perceptible third dimension in proximity and at the location of the tumor region because of the accumulation fluids (the contrast agent in the specific case). In fact, in (a) and in (b) are shown CC images calculated from T1-weighted MRI and contrast enhanced T1-weighted MRI respectively. And, as visible inside the ellipses, the visually perceptible third dimension is accentuated in (b) suggesting that the accumulation of contrast agent in the tumor region is the determinant of the magnitude of the third dimension. In other words, the contrast agent used in the T1-weighted MRI makes it possible to see clearly the visually perceptible third dimension in the $\mathrm{CC}$ image. Consistently, the CC images shown in (c), (d), (e), (f), (g), (h) and (i) were calculated from contrast enhanced T1-weighted MRI and they all show clearly the visually perceptible third dimension at the location of the tumor mass. 


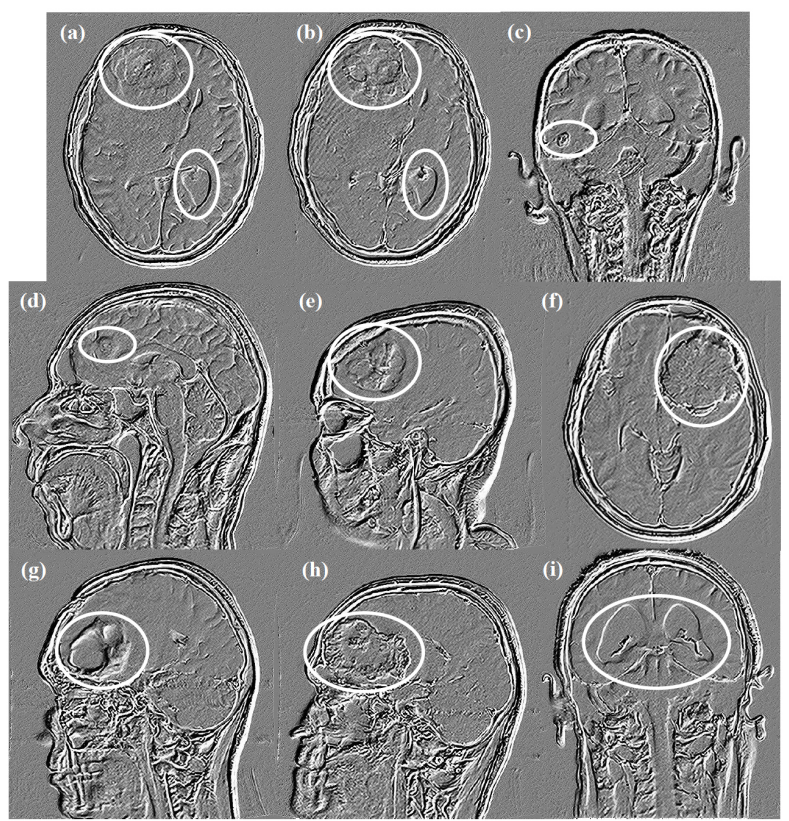

Figure 12. Classic-curvature (CC) images calculated with the bivariate cubic polynomial of equation (1) from contrast enhanced T1-weighted MRI images except for the image in (a), which is calculated from a T1-weighted MRI. The tumor regions are located inside the ellipses. (a) and (b) show the effect of the contrast agent on the classic-curvature image and the tumor pathology is oligodendroglioma. The images in (c), (d), (e), (f), (g), (h) and (i) were calculated from MRI showing the following pathologies: metastases (c), metastases from pulmonary cancer (d), anaplastic oligodendroglioma (e), meningioma (f), cystic glioblastoma $(\mathrm{g})$, glioblastoma $(\mathrm{h})$ and tumor with intraventricular extension (i). All of the classic-curvature images show the visually perceptible third dimension in the tumor regions. The Time to Echo (TE) and the Repetition Time (TR) were: $8.7 \mathrm{msec}, 550 \mathrm{msec}$ in (a); $8.7 \mathrm{msec}, 583 \mathrm{msec}$ in (b); 9 msec, $550 \mathrm{msec}$ in (c), (d), (e), (f), (g), (h) and (i).

Additionally, on the basis of the experience observed when post-processing 2D MRI images to calculate the classiccurvature and the intensity-curvature functional images, the cerebrospinal fluid and the ventricles are persistently showing the visually perceptible third dimension.

But the most resolving finding which elucidates on the meaning and the nature of the visually perceptible third dimension of the CC and the ICF images is that both of the classic-curvature and the intensity-curvature functional images, are suggested to be high pass filtered MRI signal. This manuscript has shown that the classic-curvature image (calculated when fitting the bivariate cubic polynomial to the $\mathrm{MRI}$ ), and the intensity-curvature functional image (calculated when fitting the bivariate linear function to the MRI), make it possible, when convolved with the MRI, to obtain results similar to those revealed through high pass filtering the MRI signal. Therefore, the meaning and the nature of the classic-curvature and the intensity-curvature functional images is suggested to be the high pass filtered MRI signal.

\section{Conclusion}

This manuscript provides with two main results relevant to the MRI intensity-curvature measurement approaches. The first result is the implication of the visually perceptible third dimension of the classic-curvature and the intensitycurvature functional images. The visually perceptible third dimension characteristic is such to make it possible to build medical intensity-curvature measure maps of the brain images placing the emphasis on the accumulation of fluids in the pathological human brain. The second result is the clarification of the meaning and the nature of the classic-curvature and the intensity-curvature functional, which is to act as high pass filter masks.

\section{Conflicts of Interest Disclosure}

The author declares that there is no conflict of interest statement.

\section{REFERENCES}

[1] Moseley ME, Cohen Y, Mintorovitch J, et al. Early detection of regional cerebral ischemia in cats: comparison of diffusion- and T2 weighted MRI and spectroscopy. Magnetic Resonance in Medicine 1990; 14: 330-46. PMid:2345513. http://dx.doi.org/10. 1002 /mrm. 1910140218

[2] Cutrer F, Michael A, Sorensen G, et al. Perfusion weighted imaging defects during spontaneous migrainous aura. Annals of Neurology 1998; 43: 25-31. PMid:9450765. http://dx.doi.org/10.1002 /ana. 410430108

[3] Le Bihan D, Breton E, Lallemand D, et al. MR imaging of intravoxel incoherent motions: application to diffusion and perfusion in neurologic disorders. Radiology. 1986; 161: 401-7. PMid:3763909. http://dx.doi.org/10.1148/radiology.161.2.3763909
[4] Pierpaoli C, Jezzard P, Basser PJ, et al. Diffusion tensor MR imaging of the human brain. Radiology. 1996; 201: 637-48. PMid:8939209. http://dx.doi.org/10.1148/radiology.201.3.8939209

[5] Haacke EM, Xu Y, Cheng YCN, et al. Susceptibility weighted imaging (SWI). Magnetic Resonance in Medicine. 2004; 52: 612-8. PMid:15334582. http://dx.doi.org/10.1002/mrm. 20198

[6] Haacke EM, Brown RF, Thompson M, et al. Magnetic resonance imaging: physical principles and sequence design. J. Wiley \& Sons. New York, USA, 1999.

[7] Golder W. Magnetic resonance spectroscopy in clinical oncology. Oncology Research and Treatment. 2004; 27: 304-9. http://dx.d oi.org/10.1159/000077983

[8] Shimamura AP. Toward a cognitive neuroscience of metacognition. 
Consciousness and Cognition. 2000; 9: 313-23. PMid:10924251. http://dx.doi.org/10.1006/ccog.2000.0450

[9] Nimsky C, Ganslandt O, Buchfelder M, et al. Intraoperative visualization for resection of gliomas: the role of functional neuronavigation and intraoperative 1.5T MRI. Neurological Research. 2006; 28: 482-7. PMid:16808876. http://dx.doi.org/10.1179/0161641 06X115125

[10] Ogawa S, Menon RS, Tank DW, et al. Functional brain mapping by blood oxygenation level-dependent contrast magnetic resonance imaging. A comparison of signal characteristics with a biophysical model. Biophysical Journal. 1993; 64: 803-12. http://dx. doi.o $\mathrm{rg} / 10.1016 / \mathrm{S} 0006-3495$ (93) 81441-3

[11] Cline HE, Schenck JF, Hynynen K, et al. MR-guided focused ultrasound surgery. Journal of Computer Assisted Tomography. 1992; 16: 956-65. PMid:1430448. http://dx.doi.org/10.1097/000 04728-199211000-00024

[12] Lysova AA, Koptyug IV, Sagdeev RZ, et al. Noninvasive in situ visualization of supported catalyst preparations using multinuclear magnetic resonance imaging. Journal of the American Chemical Society. 2005; 127: 11916-7. PMid:16117511. http://dx.doi.org /10.1021/ja053456v

[13] Flacke S, Fischer S, Scott MJ, et al. Novel MRI contrast agent for molecular imaging of fibrin implications for detecting vulnerable plaques. Circulation. Journal of the American Heart Association. 2001; 104: 1280-5. http://dx.doi.org/10.1161/hc3601.09 4303

[14] Ciulla C, Veljanovski D, Rechkoska Shikoska U, et al. Intensitycurvature measurement approaches for the diagnosis of magnetic resonance imaging brain tumors. Journal of Advanced Research. 2015; 6: 1045-69. http://dx.doi .org/10.1016/j.jare.2015.01.001

[15] Ciulla C, Risteski FA, Veljanovski D, et al. A compilation on the contribution of the classic-curvature and the intensity-curvature functional to the study of healthy and pathological MRI of the human brain. International Journal of Applied Pattern Recognition. In press.

[16] Ciulla C. Signal resilient to interpolation: An exploration on the approximation properties of the mathematical functions. CreateSpace Publisher. USA, 2012.

[17] Ciulla C, Rechkoska Shikoska U, Capeska Bogatinoska D, et al. On the intensity-curvature functional of the bivariate linear function: The third dimension of magnetic resonance 2D images in a tumor case study. American Journal of Signal Processing. 2014; 4: 41-8.

[18] Ciulla C, Rechkoska Shikoska U, Capeska Bogatinoska D. et al. Biomedical image processing of magnetic resonance imaging of the pathological human brain: An intensity-curvature based approach. In: A. Madevska Bogdanova, D. Gjorgjevikj (Eds): ICT Innovations, 2014 a: 56-65. Association for Information and Communication Technologies (ICT-ACT), Ohrid, Macedonia.

[19] Hajnal JV, Bryant DJ, Kasuboski L, et al. Use of fluid attenuated inversion recovery (FLAIR) pulse sequences in MRI of the human brain. Journal of Computer Assisted Tomography. 1992; 16: 8414. PMid:1430427. http://dx.doi.org/10.1097/00004728-1 99211000-00001

[20] Ciulla C. Improved signal and image interpolation in biomedical applications: The case of magnetic resonance imaging (MRI). Medical
Information Science Reference - IGI Global Publisher, Hershey, PA, USA, 2009.

[21] Ciulla C, Capeska Bogatinoska D, Risteski FA, et al. Applied computational engineering in magnetic resonance imaging: A tumor case study. International Journal of Image, Graphics and Signal Processing. 2014 b; 6: 1-9. http://dx.doi.org/10.5815/ijigsp. 20 14.07 .01

[22] Prastawa M, Bullitt E, Ho S, et al. A brain tumor segmentation framework based on outlier detection. Medical Image Analysis. 2004; 8: 275-83. PMid:15450222. http://dx.doi.org/10.1016/j.med ia.2004.06.007

[23] Daubechies I, Sweldens W. Factoring wavelet transforms into lifting steps. Journal of Fourier Analysis and Applications. 1998; 4: 247-69. http://dx.doi.org/10.1007/BF02476026

[24] Rice S. Mathematical analysis of random noise. Bell System Technical Journal. 1944; 23: 282-332. http://dx.doi.org/10.1002/j .1538-7305.1944.tb00874.x

[25] Fischi E. Inhomogeneity correction in high field magnetic resonance images: human brain imaging at 7 Tesla. Master Thesis. Universitat Politècnica de Catalunya, Barcelona, Spain. 2008.

[26] Vovk U, Pernuš F, Likar B. A review of methods for correction of intensity inhomogeneity in MRI. IEEE Transactions on Medical Imaging. 2007; 26: 405-21. PMid:17354645. http://dx.doi.org /10.1109/TMI. 2006.891486

[27] Axel L, Costantini J, Listerud J. Intensity correction in surfacecoil MR imaging. American Journal of Roentgenology. 1987; 148: 418-20. PMid:3492123. http://dx.doi.org/10.2214/ajr. 148 .2 .418

[28] Lewis EB, Fox NC. Correction of differential intensity inhomogeneity in longitudinal MR images. Neuroimage. 2004; 23: 7583. PMid:15325354. http://dx.doi.org/10.1016/j.neuroim age. 2004.04 .030

[29] Gerig G, Kubler O, Kikinis R, et al. Nonlinear anisotropic filtering of MRI data. IEEE Transactions on Medical Imaging. 1992; 11: 221-32. PMid:18218376. http://dx.doi.org/10.1109/42.141646

[30] Ashburner J, Friston KJ. Voxel-based morphometry - the methods. Neuroimage. 2000; 11: 805-21. PMid:10860804. http://dx.doi .org/10.1006/nimg. 2000.0582

[31] Coupé P, Yger P, Prima S, et al. An optimized blockwise nonlocal means denoising filter for 3-D magnetic resonance images. IEEE Transactions on Medical Imaging. 2008; 27: 425-41. PMid:18390341. http://dx.doi.org/10.1109/TMI . 2007.906087

[32] Yang J, Fan J, Ai D, et al. Brain MR image denoising for Rician noise using pre-smooth non-local means filter. BioMedical Engineering OnLine. 2015; 14: 1-20. PMid:25572487. http://dx.doi.org/1 $0.1186 / 1475-925 \mathrm{X}-14-2$

[33] Lee JE, Chung MK, Alexander AL. Evaluation of anisotropic filters for diffusion tensor imaging. In Biomedical Imaging: Nano to Macro, 3rd IEEE International Symposium on. Arlington, VA, USA. 2006: $77-8$.

[34] Manjon J, Coupé P, Buades A, et al. New methods for MRI denoising based on sparseness and self-similarity. Medical Image Analysis. 2012; 16: 18-27. PMid:21570894. http://dx.doi.org/10.1016 /j.media.2011.04.003 